Draft Version June 13, 2021

Preprint typeset using IATEX style emulateapj v. 11/26/04

\title{
QUARK-NOVAE IN MASSIVE BINARIES : A MODEL FOR DOUBLE-HUMPED, HYDROGEN-POOR, SUPERLUMINOUS SUPERNOVAE
}

\author{
Rachid Ouyed, Denis Leahy and Nico Koning \\ Department of Physics \& Astronomy, University of Calgary, 2500 University Drive NW, Calgary, AB T2N 1N4, Canada \\ Draft version June 13, 2021
}

\begin{abstract}
LSQ14bdq and SN 2006oz are super-luminous, hydrogen-poor, SNe with double-humped light curves. We show that a Quark-Nova (QN; explosive transition of the neutron star to a quark star) occurring in a massive binary, experiencing two Common Envelope (CE) phases, can quantitatively explain the light curves of LSQ14bdq and SN 2006oz. The more massive component (A) explodes first as a normal SN, yielding a Neutron Star which ejects the hydrogen envelope of the companion when the system enters its first CE phase. During the second CE phase, the NS spirals into and inflates the second He-rich CE. In the process it gains mass and triggers a Quark-Nova, outside of the CO core, leaving behind a Quark Star. The first hump in our model is the QN shock re-energizing the expanded He-rich CE. The QN occurs when the He-rich envelope is near maximum size $\left(\sim 1000 R_{\odot}\right)$ and imparts enough energy to unbind and eject the envelope. Subsequent merging of the Quark Star with the $\mathrm{CO}$ core of component B, driven by gravitational radiation, turns the Quark star to a Black Hole. The ensuing Black Hole accretion provides sufficient power for the second brighter and long lasting hump. Our model suggests a possible connection between SLSNe-I and type Ic-BL SNe which occur when the Quark Nova is triggered inside the $\mathrm{CO}$ core. We estimate the rate of QNe in massive binaries during the second CE phase to be $\sim 5 \times 10^{-5}$ of that of core-collapse SNe.

Subject headings: circumstellar matter stars: evolution stars: winds, outflows supernovae: general supernovae: individual (LSQ14bdq,SN 2006oz)
\end{abstract}

\section{INTRODUCTION}

Superluminous, hydrogen-poor, supernovae (SLSNeI) reach peak luminosities at least an order of magnitude higher than those of Type-Ia SNe and standard core-collapse SNe (e.g. Gal-Yam (2012)). They show a variety of lights curves and lack hydrogen in their spectra (Pastorello et al. (2010); Quimby et al. (2011)). They are associated with low-metallicity environments/galaxies and seem to appeal to different conditions than those of standard core-collapse and TypeIa supernovae (e.g. Chen et al. (2013); Nicholl et al. (2015a); Leloudas et al. (2015); Lunnan et al. (2015)). Popular models include powering by a millisecond spinning-down pulsar with an $\sim 10^{14} \mathrm{G}$ magnetic field Woosley (2010); Kasen\&Bildsten (2010)) and Black Hole (BH) accretion (Dexter\&Kasen (2013)). However, there is still much debate about the progenitors and the mechanism/engine powering SLSNe-I.

Here we focus on two double-humped, hydrogen-poor, SLSNe namely LSQ14bdq (Nicholl et al. $(2015 b))$ and SN 2006oz (Leloudas et al. (2012)). They have a precursor (the first hump) which is much brighter and narrower than a typical SN and a second brighter and long lasting hump. With two double-hump examples it is clear now that the first hump is not a regular SN. Thus we revise our previous model for SN 2006oz (Ouyed \& Leahy (2013)) in the context of the current work.

Here we show that a Quark-Nova (QN) occurring in a massive binary undergoing two common envelope (CE) phases offers ingredients that can account for the doublehumped light curves and other general properties of SLSNe-I such as their hydrogen-poor nature. The paper is organized as follows: in $\S 2$ we give a brief overview of the QN model and the occurrence of QNe in binary systems. In $\S 3$ we show the results of applying our model to LSQ14bdq and SN 2006oz. We provide a discussion in $\S 4$ and conclude in $\S 5$.

\section{QUARK NOVA MODEL}

The QN is the explosive transition of a neutron star (NS) to a quark star (QS) (Ouved et al. (2002); Keränen et al. (2005); see Ouyed et al. (2013a) for a review). The released energy ejects on average of $M_{\mathrm{QN}} \sim$ $10^{-3} \mathrm{M}_{\odot}$ of neutron-rich material (Keränen et al. (2005); Ouyed \& Leahy (2009); Niebergal et al. (2010)). The relativistic QN ejecta has an average Lorentz factor $\Gamma_{\mathrm{QN}} \sim 10$ and a kinetic energy, $E_{\mathrm{QN}}$, exceeding $10^{52}$ erg. The QN can occur following the explosion of a massive single star or in a binary system. The explosion is triggered when the parent NS reaches deconfinement densities in its core (e.g. Staff et al. $(2006)$ ) by becoming massive enough, via fall-back during the SN explosion, accretion from a companion or from accretion while the NS is inside a CE as considered here. The critical NS mass above which the QN explosion is triggered is defined

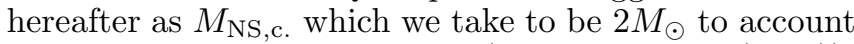
for the most massive known NS (Demorest et al. (2010)).

\subsection{Quark-Novae in single-star systems}

A dual-shock QN (dsQN) happens when the QN occurs days to weeks after the SN explosion of the progenitor star. The time delay means that the QN ejecta catches up and collides with the SN ejecta after it has expanded to large radii (Leahy \& Ouved (2008); Ouyed \& Leahy (2009)). Effectively, the QN re-energizes the extended SN ejecta causing a re-brightening of the SN. For time delays not exceeding a few days, the size of the SN ejecta is small enough that only a modest 
re-brightening results when the QN ejecta collides with the preceding SN ejecta; this yields a moderately energetic, high-velocity, SN. In this case, however, the QN model predicts that the interaction of the QN neutrons with the SN ejecta leads to unique nuclear spallation products (Ouved et al. (2011a)) which may have been observed (e.g. Laming (2014)). For longer timedelays, extreme re-brightening occurs when the two ejecta collide yielding light curves very similar to those of SLSNe (Ouved et al. 2012, 2013b; Kostka et al. 2014). For time-delays exceeding many weeks, the SN ejecta is too large and diffuse to experience any substantial re-brightening. The dsQN model has been used to fit a number of superluminous and double-humped supernovae (see http://www.quarknova.ca/LCGallery.html for a picture gallery of the fits).

\subsection{Quark-Novae in binaries}

A QN could also occur in tight binaries where the NS

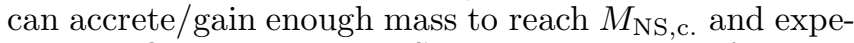
rience a QN event. The NS can accrete either from the companion overflowing its Roche Lobe Ouved \& Staff $(2013))$ or while inside a CE. QNe in binaries have proven successful in fitting properties of unusual SNe. For example, in a NS-WD system the detonation of a disrupted CO White Dwarf by the QN leads to an explosion resembling SN 2014J (what we referred to as a QNIa; Ouyed \& Staff (2013); Ouyed et al. (2014, 2015)). QNe in NS-(He)WD binaries have also been considered (Ouyed et al. (2011b.c)). In general, a QN in binaries provides:

(i) A means (the QN ejecta) to shock, and reheat the disrupted companion. The heating is on the order of $\sim 10^{9} \mathrm{~K}$ for an ejecta mass of a few solar masses and QN energy of $\sim 10^{52}$ ergs which in some cases may trigger nuclear burning.

(ii) The QS is born with a magnetic field on the order of $B_{\mathrm{QS}} \sim 10^{15} \mathrm{G}$ owing to color ferromagnetism in quark matter during the transition (Iwazaki (2005)). The spindown (SpD) power from the QN compact remnant (the QS), thus provides an additional energy source besides radio-active decay (if nuclear burning is induced by the QN shock). This additional energy source can be released on timescale $\tau_{\mathrm{QS}}=4.74$ days $P_{\mathrm{QS}, 10}^{2} B_{\mathrm{QS}, 15}^{2}$ where the QS period is given in units of 10 milliseconds and its surface magnetic field in units of $10^{15} \mathrm{G}$. The spin-down power is important in QNe-Ia where in addition to runaway thermonuclear CO burning, the spin-down energy from the QS can contribute to the light curve with interesting implications to Cosmology if some SNe-Ia are indeed QNe-Ia (Ouved et al. (2014, 2015)).

(iii) The QS could convert to a $\mathrm{BH}$ and release additional energy via BH accretion (Ouyed et al. (2011b c)). In a QN-Ia, the QS (a gravitational point mass) can slow down and trap some of the ejecta (e.g. Ouved et al. (2015)).

(iv) In general, the QN explosion (if asymmetric) can provide a kick to the QS. For a relativistic $\left(\Gamma_{\mathrm{QN}} \sim 10\right)$ QN ejecta of mass $M_{\mathrm{QN}} \sim 10^{-3} M_{\odot}$, the $\mathrm{QN}$ kick is of the order of a $100 \mathrm{~km} \mathrm{~s}^{-1}$ for a $10 \%$ asymmetry in the QN explosion.

\section{THE QUARK-NOVA IN A MASSIVE BINARY : SEQUENCE OF EVENTS}
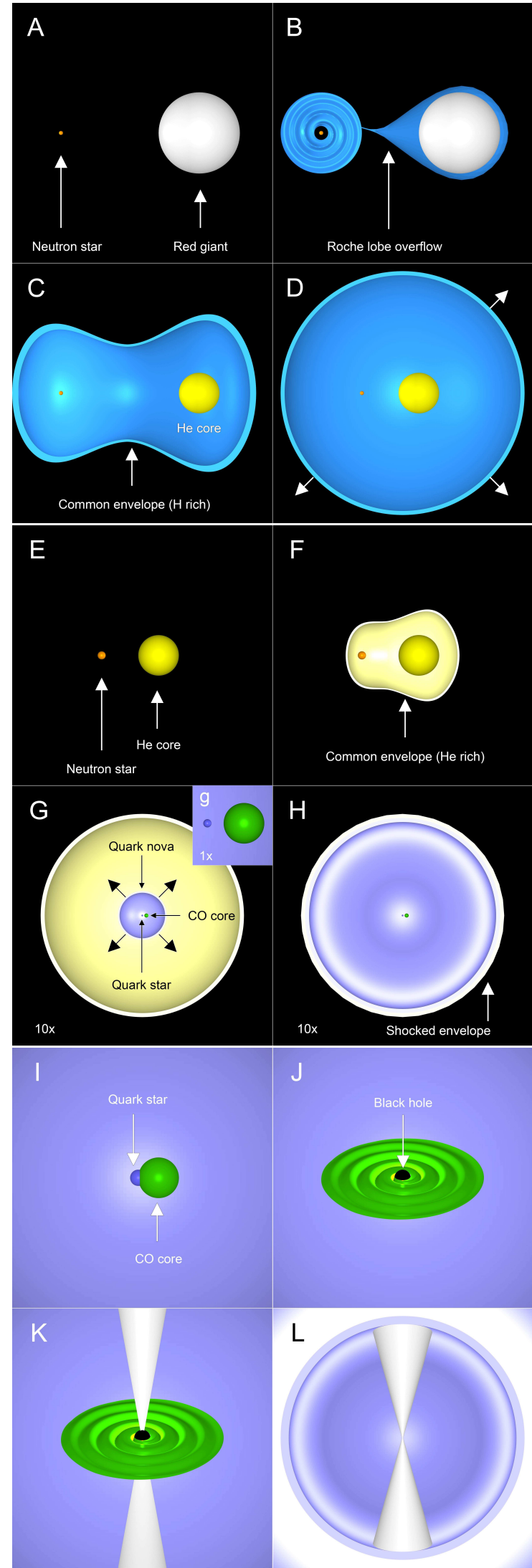

FIG. 1. - Sequence of events in a massive binary leading to the QN event (leaving behind a QS) and subsequent BH accretion of the core of the secondary companion following $\mathrm{CE}$ ejection (the $\mathrm{BH}$ forms following accretion onto the QS). The "1x" ("10x") in panel $\mathrm{g}(\mathrm{G})$ stand for 1 (10) times zoom. 
Here we consider a QN occurring in a massive binary with both components in the $\sim 20-25 M_{\odot}$ range, a mass ratio close to unity and a binary separation such that a first CE phase avoids merger (see Taam\&Ricker (2010) and Podsiadlowski (2014) for a review). We also assume a low-metallicity environment. The following sequence of events leads to the QN explosion inside the second He-rich CE (see panels in Figure 1):

(i) The more massive component (A) experiences an SN explosion leaving behind a NS with mass at birth $M_{\mathrm{NS}, \mathrm{b}} \sim 1.4 M_{\odot}$. We only consider cases where the binary survives which is possible considering a mass ratio close to unity (panel A). A typical initial state is shown in Panel "A" which consist of a NS and a Red Giant separated by $\sim 200 R_{\odot}$.

(ii) When component $\mathrm{B}$ evolves to the giant phase with a well developed Helium (He) core, the system enters the first $\mathrm{CE}$ envelope phase leading to the ejection of the hydrogen envelope of B by the in-spiralling NS (panels $\mathrm{B}, \mathrm{C}$, and D).

In the red giant phase, a component B of mass $\sim$ $20 M_{\odot}$ for example would have a radius large enough to engulf the NS (panels "C" and "D") with a $\mathrm{He}$ core mass and radius of $\sim 6.0 M_{\odot}$ and $\sim 0.5 R_{\odot}$, respectively. In comparison, a component $\mathrm{B}$ of mass $25 M_{\odot}$ would have a core mass and radius of $\sim$ $8.0 M_{\odot}$ and $\sim 0.8 R_{\odot}$, respectively (e.g. Table 7.1 of Salaris, M. \& Cassisi (2005)). In this phase, the density gradient is steep enough (e.g. Taam et al. (1978)) to reduce the accretion much below the Hoyle\&Lyttleton rate (Hoyle \& Lyttleton (1993)). Efficient CE ejection is expected with at most $\sim 0.1 M_{\odot}$ of mass accreted by the NS (e.g. MacLeod \& Ramirez-Ruiz (2015)) reaching a mass of $\sim 1.5 M_{\odot}$. Thus to reach $M_{\mathrm{NS}, \mathrm{c} .} \sim 2 M_{\odot}$, the NS would need to accrete the remaining mass during the second CE phase as described below.

(iii) As illustrated in panel E, following the first $\mathrm{CE}$ phase, one is left with a He-core-NS binary in a close orbit (e.g. Hall\& Tout (2014); see also Figure 4 in MacLeod \& Ramirez-Ruiz (2015)) with a period of a few hours. Specifically, the binary separation following ejection of the first $\mathrm{CE}$ we estimate to be of the order of $a_{\mathrm{i}} \sim 3 R_{\odot}$ for an $M_{\mathrm{NS}, \mathrm{b}} \sim 1.4 M_{\odot}$ NS and a He core of $\sim 6 M_{\odot}-8 M_{\odot}$ representative of the $20-25 M_{\odot}$ mass range of the binary components.

The secondary B continues to evolve and expands to cause a second $\mathrm{CE}$ phase (panel F). For a high enough mass ratio, a runaway mass transfer is expected once the He-rich core overflows its Roche Lobe leading to the second CE phase (e.g. Ivanova et al. (2003); Dewi et al. $(2003))^{1}$ accompanying further accretion onto the inspiralling NS.

The secondary would expand to a radius of $>3 R_{\odot}$ (thus engulfing the NS). At onset of the second $\mathrm{He} \mathrm{CE}$ phase, the envelope size is of the order of $3 R_{\odot}$ compared to about $200 R_{\odot}$ for the onset of the first CE. Thus the envelope density is much higher (a factor $(200 / 3)^{3} \sim 10^{5}$ ) in the second $\mathrm{CE}$ phase leading to much higher accretion rates. $\left(\sim 0.1 M_{\odot} \mathrm{yr}^{-1}\right.$; Brown (1995); Chevalier (1996)). Although the accretion luminosity is neutrinodominated in this regime (e.g. Chevalier (1996)), thermal energy is deposited in the CE by the photons at

\footnotetext{
${ }^{1}$ See Tauris et al. (2015) for other outcomes.
}

the Eddington rate of $L_{\text {Edd. }} \sim 4 \times 10^{38} \mathrm{erg} \mathrm{s}^{-1}$ for a $\sim 1.5 M_{\odot}$ NS. Convection transfers the deposited energy rapidly to the CE surface layers where it is radiated away. This is the self-regulating mechanism as described in Meyer \& Mever-Hofmeister (1979)) in which the frictional energy that is released during spiralling-in is transported to the surface by convection and is radiated away without causing ejection of the envelope. In our model, the $\mathrm{CE}$ ejection is caused by the QN explosion which occurs when the $\mathrm{CE}$ is near maximum size.

The maximum envelope size is defined by $4 \pi R_{\mathrm{CE}, \mathrm{max} .}^{2} \sigma T_{\mathrm{CE}, \text { eff. }}^{4}=L_{\mathrm{Edd}}$. with the envelope's effective temperature $T_{\mathrm{CE} \text {,eff. }} \sim 3000 \mathrm{~K}$ typical of fully convective stars on a Hayashi track (Hayashi (1961)). This yields $R_{\mathrm{CE}, \mathrm{max} .} \sim 1000 R_{\odot}$ at which point the envelope stops expanding (i.e. a steady energy balance is reached). For a thermal expansion speed of $c_{\mathrm{s}, \mathrm{CE}}=\sqrt{k_{\mathrm{B}} T_{\mathrm{CE}, \mathrm{eff} .} / \mu_{\mathrm{CE}} m_{\mathrm{H}}} \sim 5 \mathrm{~km} \mathrm{~s}^{-1}$, the envelope settles at its maximum radius within a few years $\left(\sim R_{\mathrm{CE}, \text { max. }} / c_{\mathrm{s}, \mathrm{CE}} \sim 4.5\right.$ years $) ; \mu_{\mathrm{CE}}=4 / 3$ is the He envelope mean atomic weight, $k_{\mathrm{B}}$ the Boltzmann constant and $m_{\mathrm{H}}$ the Hydrogen mass.

(iv) While the $\mathrm{CE}$ radius is evolving towards it maximum radius, the NS continues to in-spiral and to accrete enough mass $\left(\sim 0.4 M_{\odot}\right.$ in $\sim 4$ years $)$ to reach the critical mass $M_{\text {NS,c. }} \sim 2 M_{\odot}$ and undergo an explosive transition to a QS (panel "G"); the envelope radius at the time of the $\mathrm{QN}$ is $R_{\mathrm{CE}, \mathrm{QN}} \sim c_{\mathrm{s}, \mathrm{CE}} \times(4$ years $) \sim 900 R_{\odot}$. Interestingly the time it takes the NS to experience the QN event is close to the time it would take the $\mathrm{CE}$ to reach its maximum size; I.e. $R_{\mathrm{CE}, \mathrm{QN}} \sim R_{\mathrm{CE} \text {,max. thus offering }}$ a picture where the $\mathrm{QN}$ energy is released when the $\mathrm{CE}$ is near maximum size. This provides conditions for optimum harness of the $\sim 10^{52}$ erg of QN kinetic energy which yields the bright first hump in our model. The energy released by the QN explosion is enough to unbind the $\mathrm{CE}$ and eject it. Thus in our scenario (with a He-rich second $\mathrm{CE}$ ), the QN helps induce CE ejection. The QN shock propagating at velocity $v_{\mathrm{QN} \text {,sh. en- }}$ ergizes the $\mathrm{He} \mathrm{CE}$ (panel $\mathrm{H}$ ) and yields the first bright and short-lived hump; the corresponding initial envelope temperature is estimated from shock physics (Lang 1999), $k_{\mathrm{B}} T_{\mathrm{CE}, 0} \sim(3 / 16) \mu_{\mathrm{CE}} m_{\mathrm{H}} v_{\mathrm{QN} \text {,sh. }}^{2}$. There will be no nuclear/He burning induced by the QN shock since the conditions in the envelope (temperature and density) are below critical values.

(v) After CE ejection, one is left with a $\sim 2 M_{\odot} \mathrm{QS}$ and a CO core of mass $M_{\mathrm{CO}} \sim 2 M_{\odot}$ and radius $<0.1 R_{\text {sun }}$. The orbital separation when the QN occurs can be derived by estimating by how much the NS orbital radius has decreased when it experiences the QN event. For a Hoyle\&Lyttleton rate accretion rate, a relationship between the NS orbit and mass can be derived which is $a_{\mathrm{ON}} \sim a_{\mathrm{i}}\left(M_{\mathrm{NS}, \mathrm{i}} / M_{\mathrm{NS}, \mathrm{c} .}\right)^{\sigma}$ with $5 \leq \sigma \leq 7$ as given in Chevalier (1993) (and references therein). For $\sigma \sim 6, M_{\mathrm{NS}, \mathrm{i}} \sim 1.5 M_{\odot}$ and $M_{\mathrm{NS}, \mathrm{c} .} \sim 2 M_{\odot}$ we get $a_{\mathrm{QN}} \sim 0.18 a_{\mathrm{i}} \sim 0.54 R_{\odot} a_{\mathrm{i}, 3}$ where $a_{\mathrm{i}, 3}$ is the initial orbit separation just before the second spiral-in phase starts (i.e. immediately after the first, H-rich, $\mathrm{CE}$ has been ejected) in units of $3 R_{\odot}$.

The orbit then decays through emission of gravitational radiation. General relativity predicts that decay 
of orbital period is given by (Landau \& Lifshitz (1962))

$$
\frac{d P}{d t}=-\frac{192 \pi G^{5 / 3}}{5 c^{5}}\left(\frac{P}{2 \pi}\right)^{-5 / 3} M_{\mathrm{NS}} M_{\mathrm{CO}} M_{\mathrm{T}}^{-1 / 3},
$$

where $M_{\mathrm{T}}=M_{\mathrm{NS}}+M_{\mathrm{CO}}$ is the total mass and we assume zero orbital eccentricity. After integrating equation above and making use of Kepler's third law, this gives a decay timescale

$$
\tau_{\mathrm{GW}} \sim 9.4 \text { days } \frac{a_{\mathrm{i}, 3}^{4}}{M_{\mathrm{CO}, 2} M_{\mathrm{NS}, 2} M_{\mathrm{T}, 4}},
$$

where $M_{\mathrm{CO}, 2}, M_{\mathrm{NS}, 2}$ and $M_{\mathrm{T}, 4}$ are the CO core mass, NS mass and total mass in units of $2 M_{\odot}, 2 M_{\odot}$ and $4 M_{\odot}$, respectively. The time delay between the QN event and merging is very sensitive to the QS-CO-core separation at the start of the second spiral-in phase $\left(a_{\mathrm{i}}\right)$. To get two humps in the lightcurve, $a_{\mathrm{i}}$ can only vary by a factor of 2 .

(vi) The orbital period of the system when the QS reaches the core is of the order of a few minutes for a $\mathrm{CO}$ core of $<0.1 R_{\odot}$ in radius. Thus once the QS reaches the core, merging occurs very quickly and on timescales not exceeding a few hours (i.e. in many orbital periods). The QS merges with the CO core of B and accretes enough mass (a fraction of a solar mass) to become a black hole $(\mathrm{BH})$. The CO core is spun-up by the in-spiralling NS/QS (panel I). The bulk of the $\mathrm{CO}$ core forms a disk around the $\mathrm{BH}$ and accretes onto it (panels J, K and L) producing a luminosity $L_{\mathrm{BH}}$ to power the long lasting main hump of the light curve. The BH accretion phase is delayed from the $\mathrm{QN}$ event by the time required for the QS to reach the $\mathrm{CO}$ core $\left(\tau_{\mathrm{GW}}\right)$, turn into a $\mathrm{BH}$, merge with the core and trigger accretion. We define this time delay as $t_{\mathrm{BH}, \text { delay }} \simeq \tau_{\mathrm{GW}}$ at which point the $\mathrm{CE}$ has extended to a radius $R_{\mathrm{CE}, \mathrm{BH}}=R_{\mathrm{CE}, \mathrm{QN}}+v_{\mathrm{CE}} t_{\mathrm{BH}, \text { delay }}$ with $v_{\mathrm{CE}}=(3 / 4) v_{\mathrm{QN}, \text { sh. }}$ the CE expansion velocity induced by the QN shock; recall that $R_{\mathrm{CE}, \mathrm{QN}}$ is the $\mathrm{CE}$ radius at the onset of the QN.

\subsection{Case study : LSQ14bdq and SN 2006oz}

Table 1 shows the model's best fit parameters for LSQ14bdq. The left panel in Figure 2 2 shows the resulting fit in the g-band absolute magnitude for LSQ14bdq together with the observations from Nicholl et al. (2015b). The blue dashed curve shows the QN proper (the first hump in our model) for $M_{\mathrm{CE}}=8 M_{\odot}$ and $R_{\mathrm{CE}, \mathrm{QN}}=$ $1050 R_{\odot}$. The black dotted curve is from $\mathrm{BH}$ accretion with an initial accretion luminosity $L_{0}=3 \times 10^{44} \mathrm{erg}$ $\mathrm{s}^{-1}$. We use the prescription in Dexter\&Kasen (2013) and adopt a constant energy injection case (see their Appendix $)^{2}$. Table 1 also shows the model's best fit parameters for SN $2006 \mathrm{oz}$ with the resulting model plotted in the right panel in Figure 2 together with observations by Leloudas et al. (2012). In Table 1, $E_{\mathrm{QN}} \sim$ $E_{\mathrm{CE}, \mathrm{th}}+E_{\mathrm{CE}, \mathrm{K}}$ where $E_{\mathrm{CE} \text {,th. for simplicity was calcu- }}$ lated ignoring the contribution of radiation which needs a more detailed assessment.

\footnotetext{
2 The constant energy injection adopted for the $\mathrm{BH}$-accretion model is valid only for the early stages of $\mathrm{BH}$-accretion powered lightcurve (i.e. < 50 days; see Figure 2). A more realistic calculation should include time-dependent energy injection. We do not fit the late tail which would require fully integrated numerical light curves.
}

TABLE 1

BEST FIT PARAMETERS FOR THE LSQ14BDQ AND SN 2006 OZ LC

\begin{tabular}{|c|c|c|c|c|c|}
\hline & He-rich (i.e & second) $\mathrm{CE}$ & $\mathrm{QN}$ & BH Accre & etion \\
\hline SLSN-I & $M_{\mathrm{CE}}\left(M_{\odot}\right)$ & $R_{\mathrm{CE}, \mathrm{QN}}\left(R_{\odot}\right)$ & $v_{\mathrm{QN}, \mathrm{sh} .}(\mathrm{km} / \mathrm{s})$ & $t_{\mathrm{BH}, \text { delay }}$ (days) & $L_{0}(\mathrm{erg} / \mathrm{s})$ \\
\hline LSQ14bdq & 8 & 1050 & 30,000 & 12.5 & $3 \times 10^{44}$ \\
\hline SN $2006 \mathrm{oz}$ & 4 & 500 & 40,000 & 4.5 & $2 \times 10^{44}$ \\
\hline
\end{tabular}
IN OUR MODEL.

The duration of the accretion power in our model is given by the CO core mass $M_{\mathrm{CO}}=M_{\mathrm{B}}-\left(M_{\mathrm{H}}+M_{\mathrm{CE}}\right)$ divided by the accretion rate; $M_{\mathrm{H}}$ is the hydrogen lost by component $\mathrm{B}$ (of initial mass $M_{\mathrm{B}}$ ) during the first $\mathrm{CE}$ phase. An $L_{\mathrm{BH}} \sim 10^{44} \mathrm{erg} \mathrm{s}^{-1}$ corresponds to an accretion rate of $\sim 1 M_{\odot} \mathrm{yr}^{-1}$ for an accretion efficiency of $\sim 10^{-3}$; similar accretion rates onto the $\mathrm{BH}$ during CE evolution have been found by other studies (e.g. Armitage\&Livio (2000); see also Dexter\&Kasen (2013)). This gives the duration of the accretion powered phase of a few hundred days in agreement with the duration of the observed long lasting second humps. The power from $\mathrm{BH}$ accretion was delayed by $t_{\mathrm{BH} \text {,delay }}=12.5$ days for LSQ14bdq and 4.5 days in the case of SN $2006 \mathrm{oz}$.

\subsection{Spin-down power}

Following the QN-induced ejection of the second CE, accretion onto the QS is drastically reduced. The QS is free to spin-down $(\mathrm{SpD})$ and to release up to $E_{\mathrm{SpD}}=$ $2 \times 10^{52}$ ergs $P_{\mathrm{QS}, \mathrm{ms}}^{-2}$ ergs in rotational energy; the QS inherits its millisecond period from the NS which was spunup to millisecond equilibrium period (e.g. Tauris et al. (2012)). The QS magnetic field of $\sim 10^{15} \mathrm{G}$ (Iwazaki (2005)) imply SpD timescale of $\sim 1.1$ hours. Figure 3 shows the resulting lightcurve for SN $2006 \mathrm{oz}$ with SpD included using the prescription of Woosley (2010); Kasen\&Bildsten (2010). As can be seen SpD overwhelms the QN and when combined with BH-accretion the model yields a single broad hump. The QN shock deposits energy throughout the $\mathrm{CE}$ on short timescales and is released by the receding and cooling photosphere yielding a bright and narrow lightcurve. This is in contrast to the $\mathrm{SpD}$ (and also BH-accretion) where the energy is deposited centrally and is thus affected by the envelope's diffusion timescale which leads to a broad lightcurve.

The good fits to the two observed double-humped SLSNe (see Fig. 2) suggest that either the SpD is negligible (e.g. for $M_{\mathrm{NS}, \mathrm{b} .} \sim 1.8 M_{\odot}$ the $\mathrm{QN}$ occurs before the NS is fully recycled) or that SpD power is highly beamed.

\section{DISCUSSION}

We have shown that a $\mathrm{QN}$ in a massive binary experiencing two CE phases (in a low-metallicity environment) can account for the double-humped light curves of the SLSNe-I LSQ14bdf and SN 2006oz. We note the following:

(i) The QN is unlikely to occur in the first CE envelope since the NS can accrete at most $\sim 0.1 M_{\odot}$ (e.g. MacLeod \& Ramirez-Ruiz (2015)). However, if the NS is born massive (e.g. $\left.M_{\mathrm{NS}, \mathrm{b} .} \sim 1.9 M_{\odot}\right)$ then a $\mathrm{QN}$ can occur in the first CE as well. In this case, a "purely" Hydrogen-rich SLSN may be the outcome and possibly a double-humped lightcurve as described above if the QS also merges with the He core. 

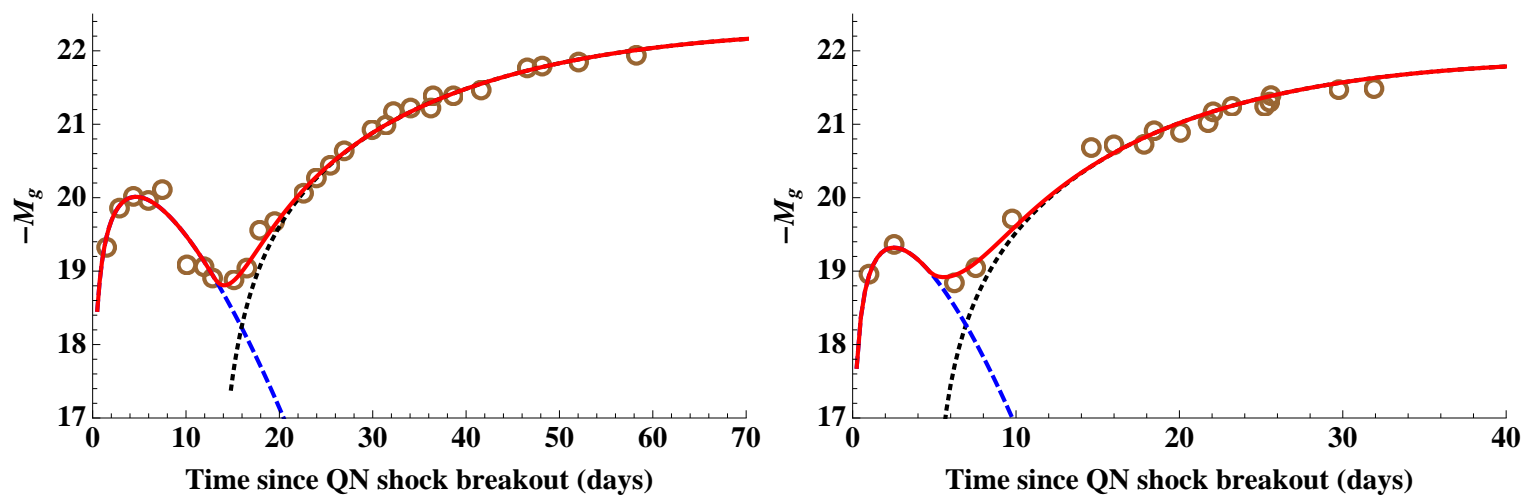

FIG. 2.- Left Panel: The QN model fit (solid line) to the g-band light curve of LSQ14bdq. The observations (the circles) are from Nicholl et al. (2015b). Right Panel: The QN model fit (solid line) to the light curve of SN 2006oz. The observations (the circles) are from Leloudas et al. (2012). In all panels, the blue dashed curve is the QN while the black dotted curve is the BH accretion.

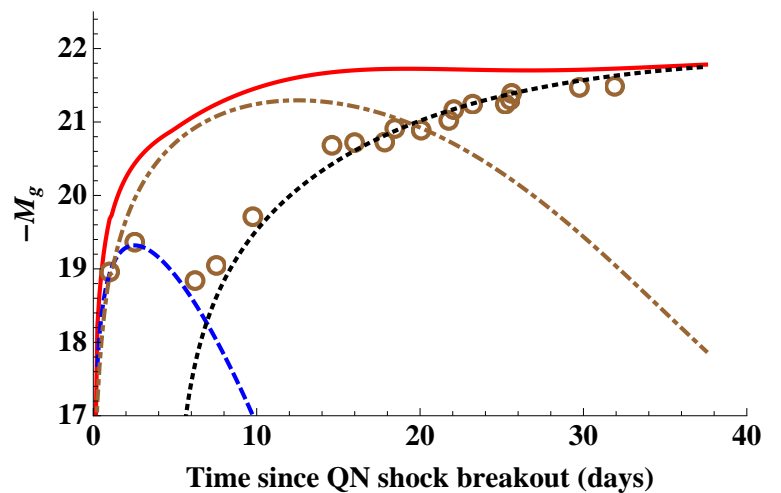

FIG. 3.- Effect of QS spin-down power (dot-dashed curve) on the g-band light curve of SN 2006oz. The combined light curve (solid line) is a single broad hump.

(ii) The QN hump has some universal features since the QN energy $\sim 10^{52} \mathrm{erg}$ and the envelope's size when the $\mathrm{QN}$ occurs $R_{\mathrm{CE}, \mathrm{QN}} \sim R_{\mathrm{CE} \text {,max. }} \sim 1000 R_{\odot}$ (within a factor of $\sim 2$ ) do not vary much. However, if the delay between the $\mathrm{QN}$ and the onset of $\mathrm{BH}$ accretion $\left(t_{\mathrm{BH} \text {,delay }}\right)$ is short, the QN hump will overlap with the BH-accretion powered hump in which case the SN should appear as a single long bright hump.

(iii) When the two humps are distinguishable, the first hump should show spectral features of an He-rich ejecta (the CE envelope) while the second longer lasting hump should also show spectral signatures of the CO-core material (the $\mathrm{BH}$ jet) when as the He envelope becomes optically thin.

(iv) In our model, the time delay between the QN (which powers the first hump) and $\mathrm{BH}$ formation and subsequent accretion (which powers the second hump) is the time it takes the QS to reach, and merge with, the $\mathrm{CO}$ core. However if the binary separation is such that $a_{\mathrm{i}}>3 R_{\odot}$ following first $\mathrm{CE}$ ejection (i.e. $a_{\mathrm{QN}}>>$ $0.5 R_{\odot}$ when the $\mathrm{QN}$ occurs) then the merger timescale can be many years making the $\mathrm{QN}$ event and the $\mathrm{BH}$ accretion as chronologically separate events. Only one hump (the QN) would be seen, yielding a type Ib SLSN. The outcome is a QS-CO-core binary which can evolve to a QS-WD or QS-NS binary depending on the conditions of the $\mathrm{CO}$ core.

(v) The BH accretion luminosity in our model of $\sim 10^{44}$ $\operatorname{erg~\mathrm {s}^{-1}}$ (i.e. $\mathrm{BH}$ accretion rates of $\sim 0.1-1 M_{\odot} \mathrm{yr}^{-1}$ at $\sim 10^{-3}$ efficiency) is low compared to the $0.01-0.1 M \odot$ $\mathrm{s}^{-1}$ accretion rates of typical long duration GRBs (e.g.
Piran (2004)). I.e. we do not expect a GRB during the $\mathrm{BH}$ in our model (see 4.1).

(vi) Here we estimate the rate of H-poor SLSNe from QNe which occur during the second CE phase of massive binaries, including both single- and double-humped events (i.e. all values of $t_{\mathrm{BH} \text {,delay }}$ ). For a Salpeter mass function (Salpeter $(1955))$ we estimate about $\sim 10 \%$ contribution to core-collapse $\mathrm{SNe}$ (CCSNe) from stars in the $\sim 20-25 M_{\odot}$ range. With about $\sim 10 \%$ of these having a companion in the $20-25 M_{\odot}$ mass range this gives $f_{\text {binary }} \sim 1 \%$ of binaries as considered here. We take an estimate of those binaries that survive the first SN explosion as $f_{\text {survival }} \sim 20 \%$ (e.g. Kalogera (1996)). In a low-metallicity environment (with weak stellar winds), we expect most of those who survived the first SN explosion to enter the first CE phase. The fraction of these binaries in a low-metallicity environment we take to be $f_{\mathrm{Z}} \sim 10 \%$ (i.e. they are more likely to occur in galaxies such as the LMC and SMC than in the Milky Way). The $f_{\mathrm{CE} .1} \sim 50 \%$ of them which survive the first CE phase (MacLeod \& Ramirez-Ruiz (2015)) will undergo a second CE phase at a high rate of $f_{\mathrm{CE}, 2} \sim 100 \%$. Finally, we assume that in $f_{\mathrm{QN}} \sim 50 \%$ of cases the QN occurs before the merger wtih the $\mathrm{CO}$ core. The overall rate is then $\sim f_{\text {binary }} \times f_{\text {survival }} \times f_{\mathrm{Z}} \times f_{\mathrm{CE}, 1} \times f_{\mathrm{CE}, 2} \times f_{\mathrm{QN}} \sim 5 \times 10^{-5}$. This is, within uncertainties, not very different from the observed rate of H-poor, type Ic, SLSNe which are also very rare with a rate of $\sim 3-8 \times 10^{-5}$ that of the corecollapse population (e.g. McCrum et al. (2015) and references therein).

\subsection{A QN inside the CO core: A possible Type Ic-BL SNe and GRB connection}

Many, but not all, long duration GRB (LGRBs) are associated with Type Ic-BL SNe (e.g. Galama (1998); Fynbo et al. (2006)). Type Ic-BL SNe are more common than LGRBs; "BL" stands for Broad-Line characterizing their $\sim 30,000-40,000 \mathrm{~km} \mathrm{~s}^{-1}$ velocities. In our model for SLSNe-I, the QN occurs during the second CE phase before the NS enters the $\mathrm{CO}$ core. It is also possible for the QN to occur after the NS enters the CO core, for instance if the accreted mass in the He-rich $\mathrm{CE}$ is not enough to drive the NS above $M_{\mathrm{NS}, \mathrm{c} .}$.

A QN inside the $\mathrm{CO}$ core leads to the following events:

(i) It provides in excess of $\sim 10^{52}$ ergs in energy which drives high-velocity CO-rich ejecta; $v_{\mathrm{CO}} \simeq$ $30,000 \mathrm{~km} \mathrm{~s}^{-1}\left(E_{\mathrm{QN}, 52} / M_{\mathrm{CO}, 2}\right)^{1 / 2}$. The compactness of 
the $\mathrm{CO}$ core means that most of the QN energy goes into the kinetic energy of the ejecta. The CO ejecta is expanding inside the $\sim 1000 R_{\odot}$ He envelope which is optically thick (optical depth of $\sim 10^{5}$ ). After the He envelope has expanded ( $\sim 10-20$ days) the high velocity CO ejecta becomes visible and should have characteristics similar to type Ic-BL SNe.

(ii) Because the QN most likely occurs off-center, the central densest parts of the $\mathrm{CO}$ core remains gravitationally bound. The remnant core would form an accretion disk around the QS. Ouyed et al. (2005) showed that accretion onto a QS can reproduce the activity (duration and intermittency) of GRBs ${ }^{3}$ from an accretion disk with a mass of a fews tenths of a solar mass. The highly collimated outflow from the QS accretion (see Ouyed et al. (2005)) has to break through the He envelope which takes of $\sim 1000 R_{\odot} / c \sim 2000 \mathrm{~s}$. If accretion lasts long enough and the jet is viewed along its axis, a QS-GRB would be seen.

(iii) The QS will likely transition to a $\mathrm{BH}$ leading to a BH-GRB in the case of high accretion rates $(\sim 0.01$ $0.1 M_{\odot} \mathrm{s}^{-1}$ ) onto the BH. The BH-GRB would immediately follow the QS-GRB. As noted above, the combined duration of the two GRBs should exceed $\sim 2000 \mathrm{~s}$ to break through the He envelope. Additionally, the QSGRB+BH-GRB would be observed only if the viewing conditions are ideal which would make this an extremely rare event. An important caveat is that to get GRBs, accretion rates from the $\mathrm{CO}$ core must be much higher than those used to fit the second hump in the lightcurves of LSQ14bdq and SN 2006oz. This suggests that GRBs require special physical conditions in this case.

\section{CONCLUSION}

\footnotetext{
3 Vogt et al. (2004) found photon emissivities in excess of $10^{50}$ erg $\mathrm{cm}^{-3} \mathrm{~s}^{-1}$ in color superconducting quark matter. The electromagnetic quark plasma frequency means that photon emissivity is suppressed for photons with energies below $\sim 23 \mathrm{MeV}$. For thermalized photons of energy $\sim 3 T_{\mathrm{QS}}$ (with $T_{\mathrm{QS}}$ being the QS temperature) this means that photon emissivity is shut-off when the QS
}

QNe should be more common in binaries with NSs (in particular if born massive) where the NS can gain sufficient mass to reach the critical mass $M_{\mathrm{NS}, \mathrm{c} .}$, to trigger quark deconfinement in their cores and go QN (Staff et al. (2006)). It is no surprise therefore that QNe should be more common in massive binaries experiencing two $\mathrm{CE}$ phases where the NS can gain the necessary mass from two mass reservoirs and go over the $M_{\mathrm{NS}, \mathrm{c}}$. limit. In our model, the QN occurs while the $\mathrm{CE}$ has expanded close to its maximum radius of $\sim 1000 R_{\odot}$ yielding a near "universal" QN (the first) hump in the resulting light-curve. The second hump attributed to $\mathrm{BH}$-accretion occurs when the $\mathrm{QS}$ turns into a $\mathrm{BH}$ as it merges with the $\mathrm{CO}$ core days following the ejection of the He-rich CE. It is also natural to expect QNe, as described here, to favor low-metallicity environments where the mass reservoirs (the two CEs) would have retained more mass over time. In high-metallicity environment, the stellar wind of component $\mathrm{B}$ can remove its envelope before it causes a second CE. The ability of the QN model in binaries to fit SLSNe in general (see http://www.quarknova.ca/LCGallery.html) and in particular double-humped hydrogen-poor SLSNe-I, as shown here, suggests that QNe may be part of binary evolution. At a more fundamental level, one of the key finding of this work is that in some cases QNe in binaries can assist with CE ejection. This can be tested by including the $\mathrm{QN}$ in simulations of common envelope evolution.

This work is funded by the Natural Sciences and Engineering Research Council of Canada.

cools below $\sim 7.7 \mathrm{MeV}$. Ouved et al. (2005) found that intermittent accretion-ejection (heating-cooling) episodes occur naturally during accretion onto the QS with an activity (variability and duration) in $\gamma$-ray emission similar to observed light curves of GRBs.

\section{REFERENCES}

Armitage, P. J. \& Livio, M. 2000, ApJ, 532, 540

Brown, G. E. 1995, ApJ, 440, 270

Chen, T.-W., Smartt, S. J., Bresolin, F., et al. 2013, ApJ, 763, L28

Chevalier, R. 1993, ApJ, 411, 36

Chevalier, R. 1996, ApJ, 459, 322

Demorest, P. B., Pennucci, T., Ransom, S. M., Roberts, M. S. E., \& Hessels, J. W. T. 2010, Nature, 467, 1081

Dewi, J. D. M., \& Pols, O. R. 2003, MNRAS, 344, 629

Dexter, J. \& Kasen, D. 2013, ApJ, 772, 30

Fynbo, J. P. U., Watson, D., Thöne, C. C., et al. 2006, Nature, 444,1047

Galama, T. J. et al. 1998, Nature, 395, 670

Gal-Yam, A. 2012, Sci, 337, 927

Hall, P. D., \& Tout, C. A. 2014, MNRAS, 444, 3209

Hayashi, C., 1961, Publ. Astron. Soc. Jap., 13, 450

Hoyle, F. \& Lyttleton, R. A. 1939, 1939, PCPS, 35, 405

Ivanova, N., Belczynski, K., Kalogera, V., Rasio, F. A., \& Taam, R. E. 2003, ApJ, 592, 475

Iwazaki, A. 2005, Phys. Rev. D, 72, 114003

Kalogera, V. 1996, ApJ, 471, 352

Kasen, D., \& Bildsten, L. 2010, ApJ, 717, 245

Keränen, P., Ouyed, R., \& Jaikumar, P. 2005, ApJ, 618, 485

Kostka, M., Koning, N., Leahy, D., Ouyed, R., \& Steffen, W. 2014, Revista Mexicana de Astronomía y Astrofísica, 50, 167

Laming, J. M. 2014, Nature, 506, 298
Landau L, \& Lifshitz E. 1962, Classical Theory of Fields. Reading, MA: Addison-Wesley

Lang, K. R. 1999, Astrophysical formulae / K.R. Lang. New York : Springer (Astronomy and astrophysics library,ISSN0941-7834)

Leahy, D., \& Ouyed, R. 2008, MNRAS, 387, 1193

Leloudas, G., Chatzopoulos, E., Dilday, B., et al. 2012, A\&A, 541, A129

Leloudas, G., Schulze, S., Krühler, T., et al. 2015, MNRAS, 449, 917

Lunnan, R., Chornock, R., Berger, E., et al. 2015, ApJ, 804, 90

MacLeod, M. \& Ramirez-Ruiz, E. 2015, ApJ, 798, L19

McCrum, M., Smartt, S. J., Rest, A., et al. 2015, MNRAS, 448, 1206

Meyer, F. \& Meyer-Hofmeister, E. 1979, A\&A, 78, 167

Nicholl, M., Smartt, S. J., Jerkstrand, A., et al. 2015a, arXiv: 1503.03310

Nicholl, M., Smartt, S. J., Jerkstrand, A., et al. 2015b, arXiv:1505.01078

Niebergal, B., Ouyed, R., \& Jaikumar, P. 2010, Phys. Rev. C, 82, 062801

Ouyed, R., Dey, J., \& Dey, M. 2002, A\&A, 390, L39

Ouyed, R., Rapp, R., \& Vogt, C. 2005, ApJ, 632, 1001

Ouyed, R., Niebergal, B., Dobler, W., \& Leahy, D. 2006, ApJ, 653, 558 
Ouyed, R., Leahy, D., \& Jaikumar, P. 2009, "Predictions for signatures of the quark-nova in superluminous supernovae" in Proceedings of the "Compact stars in the QCD phase diagram II", May 20-24, 2009, KIAA at Peking University, Beijing- P. R. China, eds. R. Ouyed \& R. Xu, http://www.slac.stanford.edu/econf/C0905202/ arXiv:0911.5424

Ouyed, R., \& Leahy, D. 2009, ApJ, 696, 562

Ouyed, R., Leahy, D., Ouyed, A., \& Jaikumar, P. 2011a, Physical Review Letters, 107, 151103

Ouyed, R., Staff, J., \& Jaikumar, P. 2011b, ApJ, 729, 60

Ouyed, R., Staff, J., \& Jaikumar, P. 2011c, ApJ, 743, 116

Ouyed, R., Kostka, M., Koning, N., Leahy, D. A., \& Steffen, W. 2012, MNRAS, 423, 1652

Ouyed, R., \& Staff, J. 2013, Research in Astronomy and Astrophysics, 13, 435

Ouyed, R., \& Leahy, D. 2013, Research in Astronomy and Astrophysics, 13, 1202

Ouyed, R., Niebergal, B., \& Jaikumar, P. 2013a, "Explosive Combustion of a Neutron Star into a Quark Star: the non-premixed scenario" in Proceedings of the Compact Stars in the QCD Phase Diagram III, arXiv:1304.8048

Ouyed, R., Koning, N., \& Leahy, D. 2013b, Research in Astronomy and Astrophysics, 13, 1463
Ouyed, R., Koning, N., Leahy, D., Staff, J. E., \& Cassidy, D. T. 2014, Research in Astronomy and Astrophysics, 14, 497

Ouyed, R., Leahy, D., Koning, N., \& Staff, J. 2015, ApJ, 801, 64 Podsiadlowski, P. 2010, New Astronomy Reviews, 54, 39

Pastorello, A., Smartt, S. J., Botticella, M. T., et al. 2010, ApJ, $724, \mathrm{~L} 16$

Piran, T. 2004, Reviews of Modern Physics, 76, 1143

Quimby, R. M., Kulkarni, S. R., Kasliwal, M. M., et al. 2011, Nature, 474, 487

Salaris, M. \& Cassisi, S., 2005 (Wiley).

Salpeter, E, 1955, ApJ, 121, 161

Staff, J. E., Ouyed, R., \& Jaikumar, P. 2006, ApJ, 645, L145

Taam, R. E., Bodenheimer, P., \& Ostriker, J. P. 1978, ApJ, 222, 269

Taam, R. E., \& Ricker, P. M. 2010, New Astronomy Reviews, 54, 65

Tauris, T. M., Langer, N., \& Kramer, M. 2012, MNRAS, 425, 1601 Tauris, T. M., Langer, N., \& Podsiadlowski, P. 2015, MNRAS, 451, 2123

Vogt, C., Rapp, R., \& Ouyed, R. 2004, Nuclear Physics A, 735, 543 Woosley, S. 2010, ApJ, 719, L204 